\title{
Open Surgery for Abdominal Aortic Aneurysm in the Era of Endovascular Repair: Comparison with Long Term Results of Endovascular Repair Using Zenith Stentgraft
}

\author{
Hisato Ito, MD, ${ }^{1}$ Takatsugu Shimono, MD, ${ }^{1}$ Hideto Shimpo, MD, ${ }^{1}$ Noriyuki Kato, $\mathrm{MD},{ }^{2}$ and Kan Takeda, $\mathrm{MD}^{2}$
}

\begin{abstract}
Objective: Our study focuses on the long term result of open surgery and endovascular abdominal aortic aneurysm repair (EVAR) using the Zenith stentgraft.

Patients and Methods: A total of 237 patients underwent elective abdominal aortic aneurysm (AAA) repair between April 1999 and December 2006. Nineteen patients underwent EVAR, whereas 218 patients underwent open surgery. The mean follow-up time for EVAR group was 73.8 \pm 49 months (range; $25-150$ months), and $69.7 \pm 46$ months (range; 1-156 months) for open surgery group.

Results: One open surgery patient $(\mathbf{1} / 218,0.46 \%)$ died of aspiration pneumonia, whereas all the EVAR patients survived the operation. Remote complications requiring reintervention occurred in 8 patients $(8 / 174,4.6 \%)$ in open surgery group. Six EVAR patients $(6 / 19,31.6 \%)$ developed late aneurysm expansion, among whom four patients $(4 / 19,21.1 \%)$ required reinterventions after 3 or more years postoperatively. The need for reintervention persisted even at 11 years after initial EVAR. There was no significant intergroup difference in late mortality.

Conclusions: There was no statistically significant intergroup difference in early and long term mortality. Complications requiring reinterventions, however, were more frequent in EVAR than in open surgery, especially in the late period. Long term follow-up is mandatory for comparison of the clinical results between open surgery and EVAR.
\end{abstract}

Keywords: aorta, aneurysm, surgery, stentgraft

\section{INTRODUCTION}

$\mathrm{C}$ urrently more than $60 \%$ of abdominal aortic aneurysm (AAA) are treated by endovascular abdominal aortic aneurysm repair (EVAR) in the United States, ${ }^{1)}$ and

${ }^{1}$ Department of Thoracic and Cardiovascular Surgery, Mie University Graduate School of Medicine, Tsu, Mie, Japan ${ }^{2}$ Department of Radiology, Mie University Graduate School of Medicine, Tsu, Mie, Japan

Received: November 29, 2012; Accepted: April 6, 2013 Corresponding author: Hisato Ito, MD. Department of Thoracic and Cardiovascular Surgery, Mie University Graduate School of Medicine, 2-174 Edobashi, Tsu, Mie 514-8507, Japan Tel: +81-59-231-5021, Fax: +81-59-231-2845

E-mail: hisato.ito@gmail.com nearly $50 \%$ in Japan, ${ }^{2)}$ and the number is still on the rise. Low perioperative mortality was observed in the published randomized control trials including EVAR trial 1, Dutch Randomised Endovascular Aneurysm Management (DREAM) trial, and Open Versus Endovascular Repair (OVER) trial., ${ }^{3,5)}$ On the contrary, there was no perioperative survival benefit of EVAR in Acarbose Cardiovascular Evaluation (ACE) trial that compared early mortality and morbidity after open surgery and EVAR in patients at low to intermediate risk. ${ }^{6}$ Open AAA repair is reported to be associated with higher operative mortality in the literatures, but some investigators emphasize that open surgery can be safely performed with low mortality even for octogenarians, ${ }^{7)}$ and for elderly patients with complex AAA anatomy in the EVAR era. ${ }^{8)}$ 
Regarding intermediate and long term survival, remote all-cause mortality was not significantly different between open AAA repair and EVAR in EVAR 1 trial and DREAM trial, and there was no intergroup difference in all-cause mortality and even in aneurysm-related death at 2 years in OVER trial. ${ }^{5)}$ On the other hand, remote aneurysmrelated mortality after EVAR for low-to-moderate risk patients was higher than open surgery in ACE trial. ${ }^{6}$ Late aneurysmal expansion and the need for reintervention are not ignorable following initially successful EVAR. As shown in DREAM trial, the cluster of reinterventions appeared in the fifth year and beyond, which suggested inferior durability of EVAR as compared to open surgery. ${ }^{9)}$ It seems that the initial benefit of EVAR disappears in the intermediate and long term because of the need for frequent, meticulous, and costly follow-up and the potential need for reinterventions, especially for patients who could tolerate open surgery.

We started to use the Zenith stentgraft system since 1999 long before it became commercially available in Japan since 2006, and our data is one of the longest follow-up of the patients who underwent EVAR using this product. This study mainly focuses on long term morbidity and mortality, and the occurrence of late reinterventions in open AAA repair and EVAR using the Zenith stentgraft.

\section{Patients and Methods}

A total of 237 patients having undergone elective AAA repair between April 1999 and December 2006 were included in this retrospective observational study after approval of the review board of Mie University Hospital. Nineteen patients underwent EVAR using the Zenith stentgraft (EVAR group), whereas 218 patients underwent conventional open AAA repair (open surgery, $\mathrm{O} / \mathrm{S}$ group). All the cases were on an elective basis and emergent cases were excluded from the study. Treatment selection was influenced by stentgraft-dependent anatomical criteria and surgical risk of open repair estimated by the surgeons. The anatomical criteria for EVAR were as follows: Adequate iliac/femoral access compatible with the required introduction systems; Non-aneurysmal infrarenal aortic segment (neck) proximal to the aneurysm with a length of at least $15 \mathrm{~mm}$, with a diameter of no greater than $28 \mathrm{~mm}$ and no less than $18 \mathrm{~mm}$ measured outer wall to outer wall, with an angle $<60^{\circ}$ relative to the long axis of the aneurysm, and with an angle $<45^{\circ}$ relative to the axis of the suprarenal aorta.
Iliac artery distal fixation site greater than $10 \mathrm{~mm}$ in length and 7.5-20 $\mathrm{mm}$ in diameter measured outer wall to outer wall. Patients with low operative risk were preferentially offered open repair even if they met the anatomical criteria for EVAR.

Hypertension, diabetes mellitus, and dyslipidemia were defined as the ones which were medically treated. Chronic obstructive pulmonary disease (COPD) was defined on the basis of pulmonary function test (forced expiratory volume $[\mathrm{FEV}] 1.0 \%<70 \%$ ) or clinical symptoms combined with the chest CT findings. Coronary artery disease (CAD) was defined as the one with a history of revascularization, and the ones that had more than $75 \%$ stenosis in the internal diameter irrespective of the need for revascularization in terms of the significance of the arteries affecting the clinical symptoms. If the patients were found to have clinically significant CAD, it was treated by percutaneous coronary intervention before surgery, or by concomitant coronary artery bypass grafting $(\mathrm{CABG})$ during open AAA repair. The mean follow-up time for EVAR group was $73.8 \pm 49$ months (range; 25-150 months), and $69.7 \pm 46$ months for $\mathrm{O} / \mathrm{S}$ group (range; 1-156 months). Forty-four out of 218 patients $(20.2 \%)$ in $\mathrm{O} / \mathrm{S}$ group were lost to follow-up within 6 months after the procedure, thus the remaining 174 patients were the subjects of the long term analysis.

\section{Statistical Analysis}

Data are presented as proportions (\%) for categorical variables and as mean \pm SD for continuous variables. Two-tailed t-test, chi-square test, and Fisher exact test were conducted as indicated to compare perioperative variables between the two groups. Probabilities of eventfree survival were obtained by Kaplan-Meier estimates for the two groups and then compared by the log-rank test. The $p$ value of 0.05 was selected as the threshold of statistical significance. Statistical analyses were performed using SPSS software version 19 for Windows (SPSS Inc, Chicago, Illinois, USA).

\section{Results}

EVAR group was older than O/S group ( $75 \pm 6.6$ vs. $71 \pm 7.9$ years old, $p<0.05$ ), and had tendency to have undergone previous abdominal surgery ( 47.1 vs. $25.0 \%$, $p=0.054)$. On the other hand, $\mathrm{O} / \mathrm{S}$ group had more CAD compared with EVAR group $(43.7 \pm 11.8 \%, p<0.05)$. There was no statistically significant difference in the rest of 
Table 1 Preoperative patient characteristics

\begin{tabular}{lccc}
\hline Characteristics & $\mathrm{S} / \mathrm{G}, \mathrm{n}=19$ & $\mathrm{O} / \mathrm{S}, \mathrm{n}=218$ & $\mathrm{p}$-value \\
\hline Age (yrs) & $75 \pm 6.6$ & $71 \pm 7.9$ & 0.038 \\
Female (\%) & 0 & 12.8 & 0.14 \\
Hypertension (\%) & 88.2 & 79.7 & 0.53 \\
Diabetes mellitus (\%) & 5.9 & 13.0 & 0.70 \\
Dyslipidemia (\%) & 35.3 & 49.3 & 0.42 \\
COPD (\%) & 23.5 & 13.8 & 0.29 \\
Creatinine (mg/dl) & 1.34 & 1.19 & 0.21 \\
History of stroke (\%) & 11.8 & 13.8 & 1.0 \\
History of abdominal surgery (\%) & 47.1 & 25.0 & 0.054 \\
CAD (\%) & 11.8 & 43.7 & 0.016 \\
Aneurysm diameter (mm) & $53 \pm 10$ & $58 \pm 13$ & 0.15 \\
\hline
\end{tabular}

COPD: chronic obstructive pulmonary disease; CAD: coronary artery disease; S/G: stentgraft group; O/S: open surgery group

Table 2 Operative data of open AAA repair

\begin{tabular}{llc}
\hline & & $\mathrm{n}=218(\%)$ \\
\hline Approach & Median laparotomy & $198(90.8)$ \\
& Retroperitoneal approach & $10(4.6)$ \\
& Mini-median laparotomy & $8(3.7)$ \\
& Spiral incision & $2(0.9)$ \\
Prosthesis & Straight graft & $47(21.6)$ \\
Reconstructed branches & Bifurcation graft & $171(78.4)$ \\
& IMA & $22(10)$ \\
& IIA & $21(9.6)$ \\
& Renal artery & $3(1.4)$ \\
& CABG & $2(0.9)$ \\
& OPCAB & $5(2.29)$ \\
& Removal of a S/G & $3(1.38)$ \\
& TEVAR & $1(0.46)$ \\
& Partial resection of the A/G & $2(0.9)$ \\
& Jejunostomy & $1(0.46)$ \\
\hline
\end{tabular}

AAA: abdominal aortic aneurysm; IMA: inferior mesenteric artery; IIA: internal iliac artery; CABG: coronary artery bypass grafting; OPCAB: off-pump coronary artery bypass grafting; $\mathrm{S} / \mathrm{G}$ : stentgraft; TEVAR: thoracic endovascular aneurysm repair; A/G: adrenal gland

the preoperative characteristics including maximal aneurysm diameter (Table 1). A 79- year-old man in O/S group $(1 / 218,0.46 \%)$ died of postoperative aspiration pneumonia, whereas all the patients in EVAR group survived the procedure. Concomitant procedures were performed for the patients in $\mathrm{O} / \mathrm{S}$ group, which were as follows: $\mathrm{CABG}$ in 2; off-pump CABG (OPCAB) in 5; TEVAR for thoracic descending aortic aneurysm in one; partial resection of the adrenal glands in two; and jejunostomy in one patient. Three patients had a history of previous EVAR and underwent open AAA repair with removal of the previously implanted stentgrafts. The procedural data are summarized in Table 2. Most of the patients underwent open surgery through median laparotomy. Limited median laparotomy with incision of $10 \mathrm{~cm}$ or less was applied in eight patients, and retroperitoneal approach was used in 10 patients. Spiral incision with limited thoracotomy was performed in two patients with pararenal AAA requiring suprarenal aortic cross clamp especially when the superior mesenteric artery (SMA) and the renal arteries were in close vicinity. Regarding reconstruction of the visceral arteries, the inferior mesenteric artery (IMA), the renal artery, and the internal iliac arteries were reconstructed in 22,3 , and 21 patients, respectively. There were no clinically apparent cardiac complications despite the fact that nearly half of the patient in $\mathrm{O} / \mathrm{S}$ group had CAD. There was only one patient in $\mathrm{O} / \mathrm{S}$ group who developed renal failure requiring permanent hemodialysis after surgery, 
Table 3 Early and late outcomes

\begin{tabular}{lccc}
\hline & $\mathrm{S} / \mathrm{G}(\mathrm{n}=19)$ & $\mathrm{O} / \mathrm{S}(\mathrm{n}=218)$ \\
\hline $\begin{array}{l}\text { In-hospital mortality } \\
\text { Operative complications }\end{array}$ & $0 / 19$ & & $1 / 218(0.45 \%)$ \\
$\quad$ Brachial artery pseudoaneurysm & $2 / 19(10.5 \%)$ & Wound dehiscence & $3 / 218(1.37 \%)$ \\
Femoral artery injury & 1 & Bowel obstruction & 1 \\
& 1 & EIA dissection & 1 \\
Remote complications & $4 / 19(21.1 \%)$ & & 1 \\
Type II EL & 1 (coiling at 38 months) & Graft leg occlusion & 2 (F-F bypass) \\
Type Ib EL & 1 (EVAR at 41 months) & Graft infection & 1 (graft removal, A-F bypass) \\
Type II EL & 1 (open surgey at 43 months) & Bowel strangulation & 1 (open surgery) \\
Type Ib EL & 1 (EVAR at 133 months) & Abdominal incisional hernia & 3 (surgery) \\
& & Aorto-enteric fistula & 1 (EVAR) \\
\hline
\end{tabular}

S/G: stent graft group; O/S: open surgery group; EIA: external iliac artery; EL: endoleak; EVAR: endovascular aneurysm repair; F-F bypass: femorofemoral crossover bypass; A-F bypass: axillofemoral bypass

which was even anticipated preoperatively considering his stage 4 chronic renal failure. With regard to in-hospital complications requiring surgical intervention, there were three patients in $\mathrm{O} / \mathrm{S}$ group $(3 / 218,1.37 \%)$, and two patients in EVAR group (2/19, 10.5\%). In $\mathrm{O} / \mathrm{S}$ group, one patient had abdominal wound dehiscence, another patient had bowel obstruction, and the third patient had left external iliac artery dissection that was found immediately after arrival in the intensive care unit (ICU) and required revision of the distal anastomosis. In EVAR group one patient had right brachial artery pseudoaneurysm, and the other patient had right femoral artery injury. These complications were arterial access route-related.

Remote complications included eight patients in O/S group (8/174, 4.6\%), which were as follows: unilateral occlusion of the bifurcated graft leg that required femorofemoral crossover bypass in two patients; graft infection requiring removal of the previous graft and axillofemoral bypass in one patient; bowel strangulation in one patient; abdominal incisional hernia in three patients; and aorto-enteric fistula in one patient, which resulted in successful emergent endovascular stentgraft placement. None of these patients died of the complications. On the other hand, there were six patients in EVAR group $(6 / 19,31.6 \%)$ that developed aneurysmal dilatation due to endoleak in the long term. Among them, four patients $(4 / 19,21.1 \%)$ required reintervention which were as follows: Two patients had type II endoleak, which resulted in unsuccessful coil embolization of the lumber arteries in one patient at 38 months postoperatively, and hemostasis of the back bleeding from the

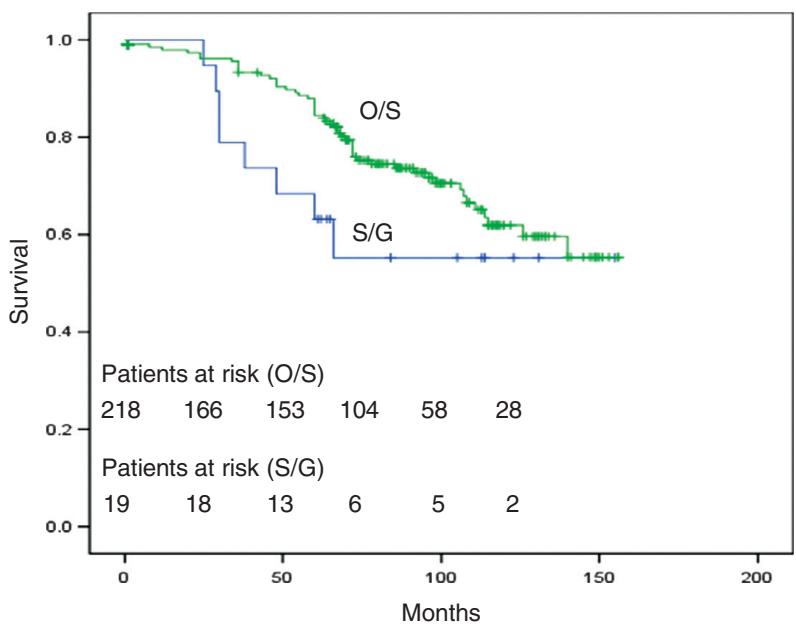

Fig. 1 Five-year survival rate was $84.5 \%$ in open surgery group and $63.2 \%$ in stent graft group, which was not statistically significantly different. (Log-rank, $p=0.13$ ). $\mathrm{O} / \mathrm{S}$ : open surgery group; $\mathrm{S} / \mathrm{G}$ : stentgraft group

lumber artery by open surgery in the other patient at 43 months postoperatively. The former patient has been followed-up on an outpatient basis since.

Two other patients developed type Ib endoleak and they underwent successful additional stentgraft placement (Table 3). One patient underwent left stentgraft leg extension to the external iliac artery at 41 months. An $18 \mathrm{~mm}$ ipsilateral leg was placed in the left common iliac artery measured $16 \mathrm{~mm}$ in diameter in the initial EVAR, where late inappropriate apposition seemed to have occurred. The patient also had $46 \mathrm{~mm}$ right common iliac artery aneurysm contiguous with the main AAA, 
which made the aortic bifurcation $58 \mathrm{~mm}$ wide. The other patient had the stentgraft legs positioned in the non-aneurysmal common iliac arteries in the initial EVAR, but he later developed a right internal iliac artery aneurysm, which seemed to have caused malapposition of the stentgraft leg. The patient underwent right stentgraft leg extension to the external iliac artery at 133 months after the initial procedure. In all the six cases with remote aneurysmal dilatation, some endoleaks were observed at the end of the initial procedure or on $\mathrm{CT}$ at discharge, most of which were considered insignificant and assumed self-limiting in the long term, though. No late limb occlusion was observed in EVAR group.

Long-term survival rate was compared by the log-rank test, and there was no statistically significant intergroup difference $(p=0.13)$. There was no abdominal aortarelated death in both groups in the long term (Fig. 1).

\section{Discussion}

The present study shows the long term results of open AAA repair and EVAR using the Zenith stentgraft performed at a single institution. The Zenith stentgraft system was approved for clinical use by the Labor and Welfare Ministry in Japan in 2006, whereas we started to use it as part of a clinical trial in 1999, hence our result is one of the limited number of studies that evaluate the long term result of the Zenith stentgraft in Japan. Main findings of the present study were that early mortality and morbidity rate was not statistically significantly different between the EVAR and open repair groups, and that there was no statistically significant intergroup difference in long term survival. Yet EVAR was associated with a significantly higher rate of late graft-related complications and reinterventions than was open surgery.

There was one perioperative death in the present study due to aspiration pneumonia in an elderly patient, but the overall early result of open repair was satisfactory. One possible reason for the good operative result was that there was no clinically apparent cardiac events after surgery because clinically significant $\mathrm{CAD}$ was revascularized before or during AAA repair.

Regarding intermediate and long term survival, remote all-cause mortality was not significantly different between the two treatments in EVAR trial 1 and DREAM trial, and there was no intergroup difference in aneurysmrelated death at 2 years in OVER trial. ${ }^{5)}$ Likewise, there was no long term survival advantage of EVAR in the present study, there were no aneurysm-related or aorta- related deaths, though. Advanced age of the EVAR patients probably accounts for this finding.

With regard to remote complications, there were eight patients who had late complications after open AAA repair in our study $(8 / 174,4.6 \%)$, which was much less frequent than was seen in DREAM trial $(20 \%))^{4}$ ) Another important finding in the present study was that EVAR was associated with a significantly higher rate of late graft-related complications and reinterventions than was open surgery. We observed six patients (31.6\%) who had aneurysm expansion late after the initial procedure, among whom four patients $(21.1 \%)$ required reinterventions because of continuing aneurysm expansion due to type II or type Ib endoleak beyond 3 years postoperatively. The need for reintervention persisted even at 11 years after initial EVAR in our study. Nevala and colleagues reported approximately $30 \%$ of the patient who underwent EVAR with the Zenith stentgraft required reintervention. ${ }^{10)}$ The authors focused on type II endoleak following Zenith stentgraft placement in another report, in which type II endoleak occurred in $22 \%$ of the patients, and reintervention was required in $6.4 \%$ of the entire cohort. They performed translumber or transarterial embolization with success rate of approximately $38 \%$, and concluded the results of percutaneous reinterventions for type II endoleak were unsatisfactory. ${ }^{11)}$ Likewise, endovascular reintervention was successful in two out of four patients, unsuccessful in one patient, and one patient required open surgery in the present study. In DREAM trial, long term survival rate was similar between open repair and endovascular repair groups, whereas the need for reintervention was more frequent in EVAR group than that in open repair group. Interestingly, the cluster of reinterventions appeared in the fifth year and beyond after EVAR, which suggested inferior durability of EVAR as compared to open surgery. ${ }^{9}{ }^{9}$ These data also coincide with the present study in terms of the significant increase of reintervention rate not in the early years, but several years after the procedure. It seems that the initial benefit of EVAR disappears in the intermediate and long term because of the need for frequent, meticulous, and costly follow-up and the potential need for reinterventions. Moreover, late open conversion after EVAR is reported to be associated with significant morbidity and mortality if explant of the stentgraft is inevitable, ${ }^{12)}$ and under this circumstance, suprarenal, and even supraceliac aortic clamping is often required. ${ }^{13)}$

Gupta and colleagues reported similar perioperative mortality after open surgery and EVAR in patients 
younger than 60 years of age, ${ }^{14)}$ and ACE trial resulted in the same early results, yet higher risk of late reintervention and aneurysm-related mortality in EVAR for lowto-moderate risk patients. ${ }^{\text {) }}$ Our results and the literatures cast a word of caution that EVAR should not be chosen for young patients without consideration simply because the anatomy of the aneurysm is suitable for EVAR when open repair can be safely performed with low mortality in skillful hands. On the other hand, one could argue that endovascular devices and techniques have been undergoing further development that could mitigate the remote risk of reintervention. Further investigation is to be waited on long term results of EVAR with third generation stentgrafts performed in the current era.

In conclusion, there was no statistically significant intergroup difference in early and long term mortality. Open surgery can be safely performed with low operative mortality and is durable. Complications requiring reinterventions, however, were more frequent in EVAR than in open surgery, especially in the far remote period. Long term follow-up is mandatory for a comparison of the clinical results between open AAA repair and EVAR.

\section{Study Limitations}

First, this study is a retrospective and observational study which can involve several biases including case selection bias. Second, the number of patients with EVAR was small since patient cohort was limited to one type of stentgraft, which first became commercially available in Japan and has the longest history as a commercial product. Although not dealt with or discussed in this article, other commercially available second generation stentgrafts also has similar issues in our experience, as partly shown in our open surgery result, which includes three patients who had undergone initial EVAR and required stentgraft removal during the late reintervention. Third, approximately $20 \%$ of the open AAA repair patients were lost to follow-up before 6 months after the procedure, which might have underscored late complication rate in this group.

\section{Disclosure Statement}

None.

\section{REFERENCES}

1) Kent KC. Endovascular aneurysm repair-is it durable? N Engl J Med 2010; 362: 1930-1.

2) Kato N, Inouchi M, Chino S. [Endovascular therapy of abdominal aortic aneurysms: present and future status in Japan]. Nihon Geka Gakkai Zasshi 2011; 112: 32-7.

3) EVAR trial participants. Endovascular aneurysm repair versus open repair in patients with abdominal aortic aneurysm (EVAR trial 1): randomized controlled trial. Lancet. 2005; 365: 2179-86.

4) Prinssen M, Verhoeven EL, Buth J, et al. A randomized trial comparing conventional and endovascular repair of abdominal aortic aneurysms. N Engl J Med 2004; 351: 1607-18.

5) Lederle FA, Freischlag JA, Kyriakides TC, et al. Outcomes following endovascular vs open repair of abdominal aortic aneurysm: a randomized trial. JAMA 2009; 302: 1535-42.

6) Becquemin JP, Pillet JC, Lescalie F, et al. A randomized controlled trial of endovascular aneurysm repair versus open surgery for abdominal aortic aneurysms in low- to moderate-risk patients. J Vasc Surg 2011; 53: $1167-73$.

7) Woo EY, Ullery BW, Carpenter JP, et al. Open abdominal aortic aneurysm repair is feasible and can be done with excellent results in octogenarians. J Vasc Surg 2011; 53: 278-84.

8) Hiromatsu S, Sakashita H, Okazaki T, et al. Perioperative outcomes for elective open abdominal aortic aneurysm repair since the adoption of endovascular grafting procedures. Eur J Vasc Endovasc Surg 2011; 42: 178-84.

9) De Bruin JL, Baas AF, Buth J, et al. Long-term outcome of open or endovascular repair of abdominal aortic aneurysm. N Engl J Med 2010; 362: 1881-9.

10) Nevala T, Perälä J, Aho P, et al. Outcome of symptomatic, unruptured abdominal aortic aneurysms after endovascular repair with the Zenith stent-graft system. Scand Cardiovasc J 2008; 42: 178-81.

11) Nevala $T$, Biancari F, Manninen $H$, et al. Type II endoleak after endovascular repair of abdominal aortic aneurysm: effectiveness of embolization. Cardiovasc Intervent Radiol 2010; 33: 278-84.

12) Chaar CI, Eid R, Park T, et al. Delayed open conversions after endovascular abdominal aortic aneurysm repair. J Vasc Surg 2012; 55: 1562-9.e1.

13) Forbes TL, Harrington DM, Harris JR, et al. Late conversion of endovascular to open repair of abdominal aortic aneurysms. Can J Surg 2012; 55: 254-8.

14) Gupta PK, Ramanan B, Lynch TG, et al. Endovascular repair of abdominal aortic aneurysm does not improve early survival versus open repair in patients younger than 60 years. Eur J Vasc Endovasc Surg 2012; 43: 506-12. 